R. J. Cohen and W. T. Sullivan, III, eds.

\title{
Fixed and Mobile RFI Search Facilities at Medicina
}

\author{
S. Montebugnoli, G. Tomassetti, C. Bortolotti and M. Roma \\ Institute of Radio Astronomy, CNR, Bologna, Italy
}

\begin{abstract}
Fixed and mobile RFI search facilities at the Medicina radio astronomy station are described. A complex system of wide-band antennas that can be steered, has been designed and installed on the top of a 25-m high tower and connected with a wide-band sweeping receiver system. At present it works from $0.08 \mathrm{GHz}$ up to $2.5 \mathrm{GHz}$ in continuous mode. Low-noise front ends are used in the radio astronomy bands. A mobile system, equipped with a similar receiving system, has been designed to facilitate the interfering transmitter's position localization.
\end{abstract}

The Medicina radio telescope station comprises two radio telescopes: the Northern Cross 610-m $\times 564-\mathrm{m}$ "T"-shaped antenna and the 32-m VLBI dish (Figure 1). Due to both the high sensitivity and the pollution situation in the radio bands, these systems are very vulnerable to radio frequency interference (RFI). In order to control the frequency bands allocated to radio astronomy, a 25-m high tower, with a proper antenna system and a completely equipped van have been set up since the beginning of the 1980s. The RFI situation control is performed through both the tower and the van (Figure 2).

The antenna system is installed on a tower (25-m height) and it is completely adjustable, over a $360^{\circ}$ range in the horizontal plane, from a remote control room. The antenna position is controlled via a 12-bit absolute optical encoder. Both antennas and front ends are user-selectable and depend on the used band. In Figure 2 the overall multi-band antenna system is shown. It is remotely adjustable at a programmable speed with a steering error of about $0.1^{\circ}$. The received signals are sent to a 40 -m distant control room (Figure 3). Very low-loss coaxial cable has been widely employed.

The operating frequencies of the tower system are:

- 80/500 MHz: both linear polarizations are available with two log-periodic antennas. The FM is attenuated with a proper filter band.

- 500/1000 MHz: both linear polarizations are available with two log-periodic antennas.

- $325 \pm 8 \mathrm{MHz}$ : double yagi system for each polarization. Low-noise frontends are used.

- $408 \pm 8 \mathrm{MHz}$ : double yagi system for each polarization. Low-noise frontends are used.

- $608 \pm 4 \mathrm{MHz}$ : double yagi system for each polarization. Low-noise frontends are used. 

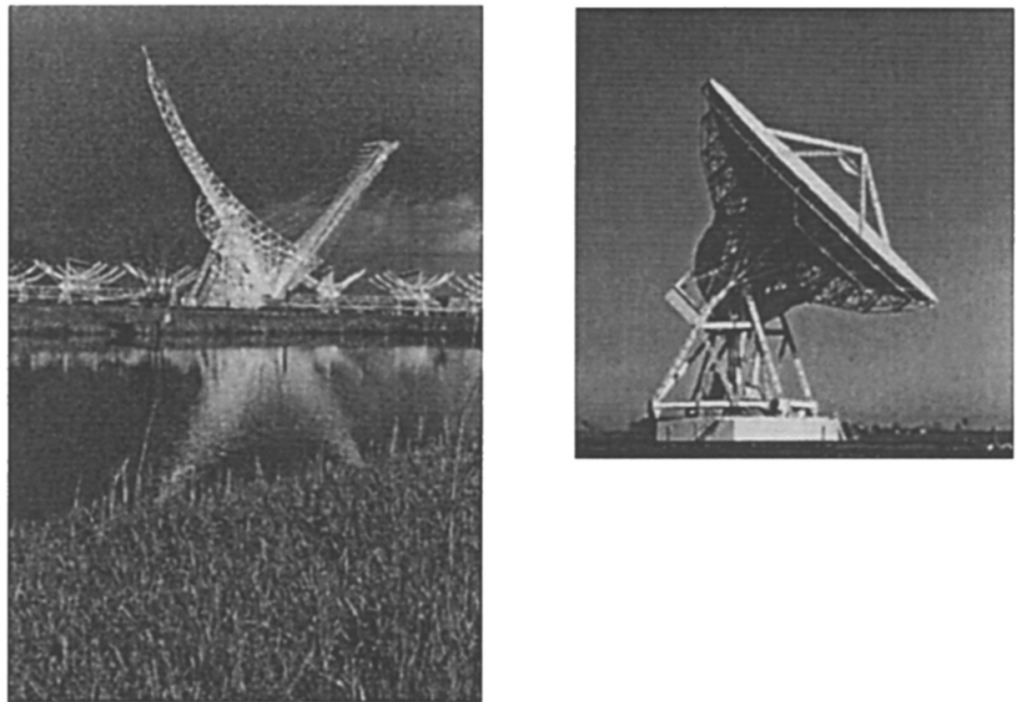

Figure 1. The Medicina radio telescopes. Left: The Cross antenna; Right: The 32-m VLBI antenna
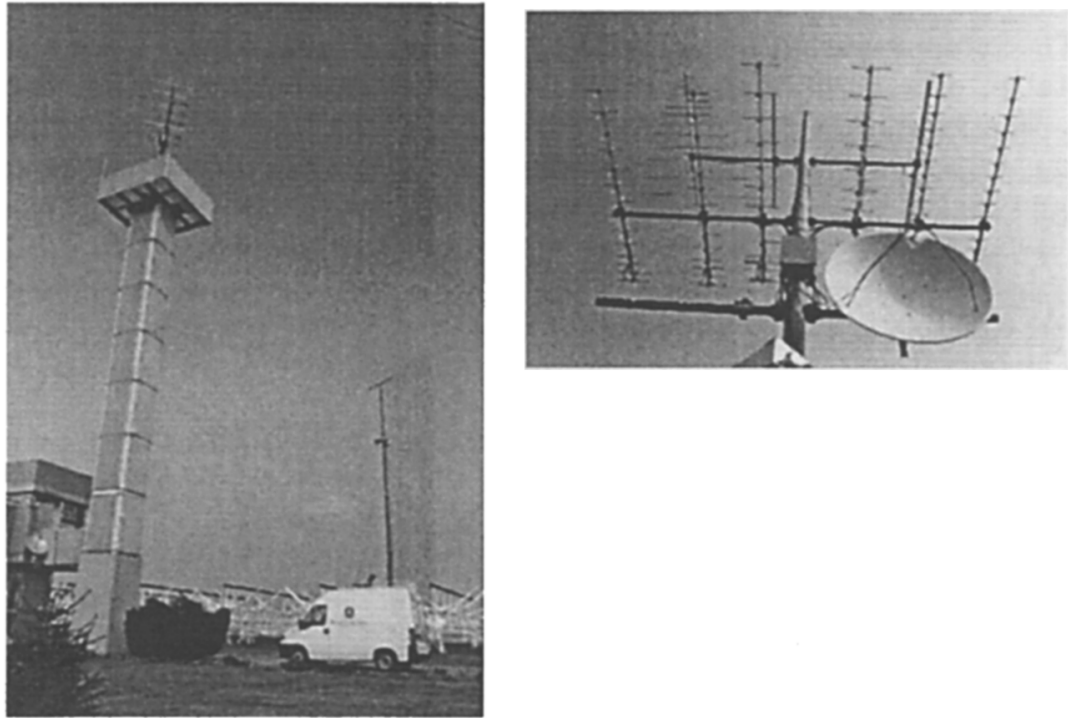

Figure 2. Left: The 25-m RFI tower at Medicina; Right: The multiband system installed on the RFI tower. 


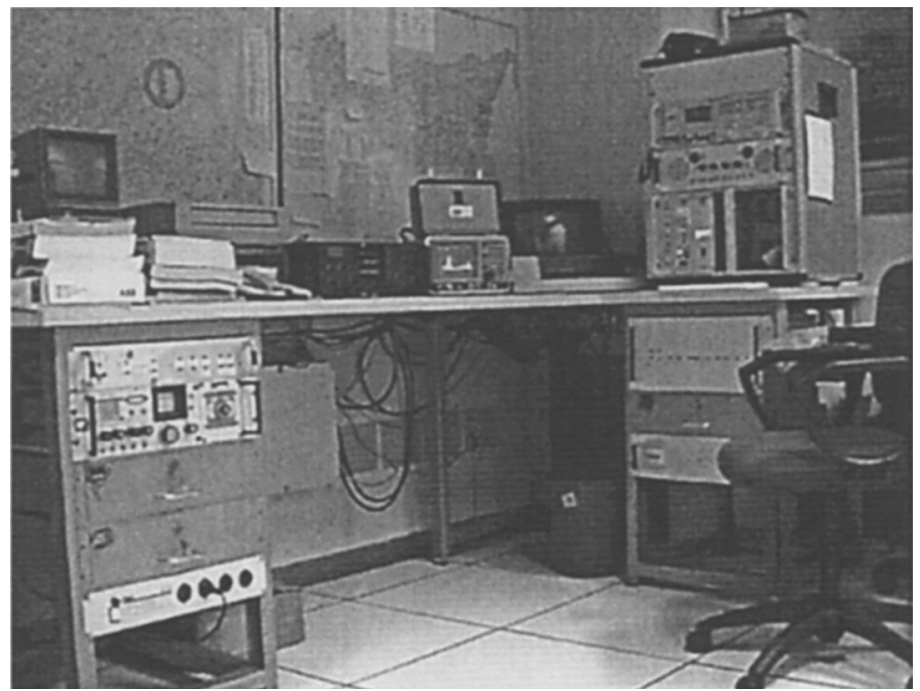

Figure 3. RFI control room at Medicina radio astronomy station.

- $1 / 2.5 \mathrm{GHz}: 1.2-\mathrm{m}$ dish with $45^{\circ}$ linear polarized feed. A low-noise frontend is used. A future upgrade up to $12 \mathrm{GHz}$ is planned.

The van is a fully equipped mobile RFI monitoring system and a remotely controlled mast extendable up to $12 \mathrm{~m}$ is installed on it (for antenna support and orientation). The antenna position is controlled via an incremental optical encoder with programmable offset. A completely autonomous power supply source is installed to allow operations in any situation. A GPS receiver and communication facilities have been installed on board. The operating frequencies of the mobile facility are:

- $310 / 430 \mathrm{MHz}$ : Log-periodic antenna (linear polarization)

- 603/611 MHz : Log-periodic antenna (linear polarization)

- 1/2.5 GHz : Double-ridged horn or Log periodic antenna (linear polarization)

- 2/18 GHz (four bands): Double-ridged horn (linear polarization)

In Figures 4 and 5, rear views of the van are shown. In the interior view the HP8562A 2 6.5-GHz Spectrum Analyzer, the pneumatic extendable mast, the receiver and the Pentium II PC are visible. The rear sides of the instruments are accessible for connections and/or expansion. These instruments are installed in special shock-absorber crates. All the frontend blocks (tower and van) are fully equipped with low-noise amplifiers and good shaped filters while a coaxial relay set allows the operator to chose the proper antenna. 


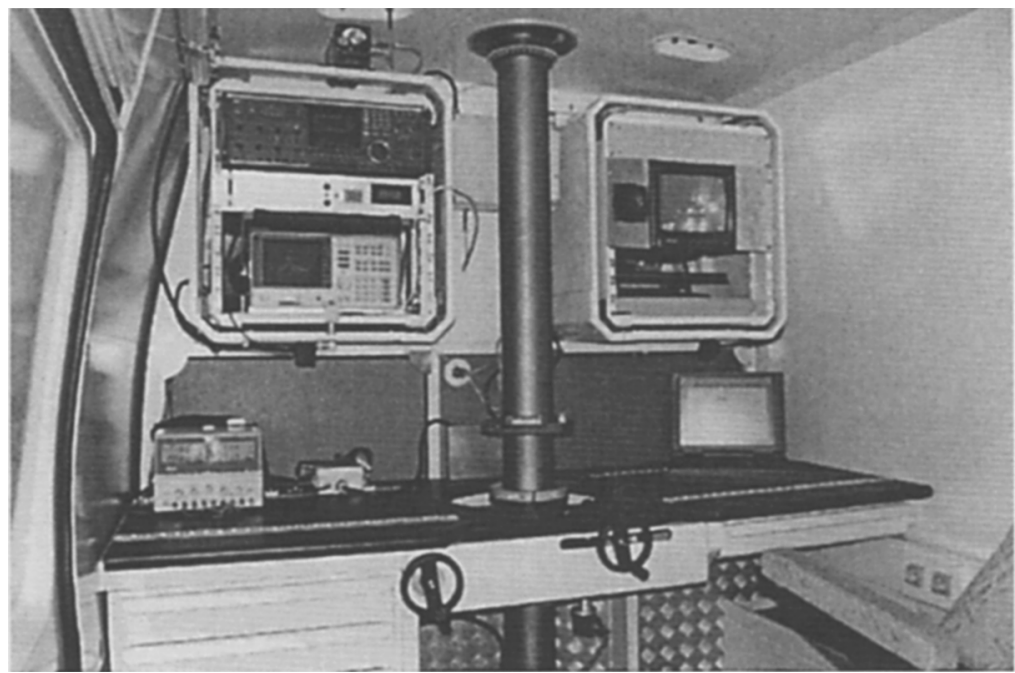

Figure 4. Medicina RFI van, interior view.

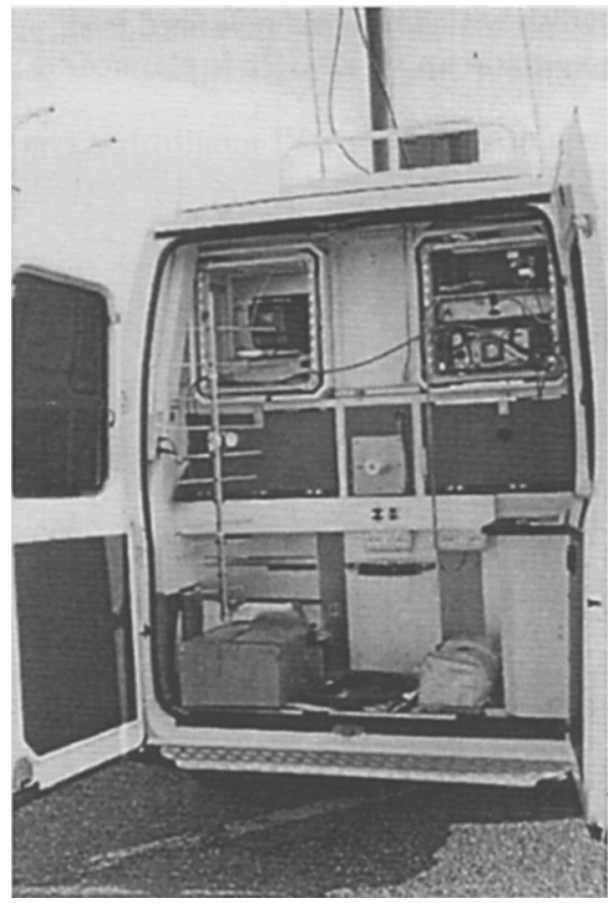

Figure 5. Medicina RFI van, rear view. 